\title{
Responsive Knit: the evolution of a programmable material system
}

\author{
SCOTT Jane \\ The University of Leeds \\ j.c.scott@leeds.ac.uk \\ doi: $10.21606 /$ dma.2018.566
}

\begin{abstract}
Programmable knitting presents a new class of behaving textiles, responsive to environmental stimuli and programmed to change in shape as humidity levels in the environment increase. It is a hierarchical system that exploits the inherent functionality of textile fibres, yarns and fabrics to integrate shape change behaviour into the intrinsic structure of the material. The research applies a biomimicry methodology, with insight derived from the structural organisation of plant materials; specifically, the control of hygromorphic actuation for seed dispersal. This biological model has produced transferable principles for application to responsive textiles and it has been critical to the success of the research. But how can this research advance thinking on the design potential of programmable materials? This paper explores how the complex hierarchies that exist within textiles can be used to engineer a unique class of programmable systems. This challenges conventional smart interfaces that rely on mediated responses via electronic control. Instead this paper demonstrates how an alternative approach informed by biomimicry can generate a new class of smart-natural materials.
\end{abstract}

programmable materials, knitting, biomimicry, the responsive environment

\section{Introduction}

Digital fabrication tools have transformed the way designers are thinking about materials. The ability to manufacture directly from computational models using 3D print technologies and robotics have presented a challenge to reconsider the potential of the underlying materials themselves (Tibbits, 2017:14). New classes of materials are emerging that have been designed with the ability to sense and respond to a range of stimuli, producing intelligent responses to changes in heat, light, moisture or pollution levels in the environment. By directly connecting with environmental stimuli, these materials have the ability to act as physical sensing systems, reducing the need for mediated systems using electronic control. Whilst many of these active, programmable materials are composed of 
smart and synthetic materials, there is class of these materials that utilise the inherent properties of conventional materials like wood, metals and textiles.

This research positions knitted fabric as a unique programmable material system with which to engage with the responsive environment. The research question asks how programmable knitting can advance the field of programmable materials for the responsive environment. The aim of the research paper is to analyse knit production processes from a hierarchical perspective, highlighting how this can be used to develop programmable materials. In addition, the paper identifies the significance of a textiles logic and identifies how it differs from other advanced manufacturing production.

Whilst smart textiles conventionally utilise either smart synthetic materials or additional electronic components to generate shape change functionality in fabrics, this research discusses how the application of a biomimicry methodology has informed fabric development. Through mapping the hierarchies that exists in plant materials against those fundamental to knitted fabrics, the ability to engineer shape change functionality directly into the fabric during production has been established (Scott, 2015). By positioning the environmental stimuli at the centre of the research, constituent materials engage directly with the stimulus. In so doing this research uses knitted fabrics, composed of $100 \%$ natural materials as a shape-changing interface to reflect and respond to the dynamic nature of the responsive environment (Scott, 2015).

The significance of the research is how the underlying principles of knit design and technology have been exploited to produce a unique behaving textile. Whilst other programmable materials utilise 3D print technologies or CNC tooling, this system originates from an understanding of textile fibres, yarns and fabric structures, and exploits the complex hierarchies inherent to knitted fabric as a foundation for the programmable material system. The application of hierarchy in the design of programmable knitting sets an important precedent with implications for the design of alternative programmable material systems.

\section{Context}

Interior and exterior space can be digitally mapped according to parameters including heat, light and moisture in order to understand the underlying patterns of energy flow. The interior environment is subject to the same physical elements as those which create weather systems outdoors. In a contained space hot air rises and cool air sinks. Water evaporates into the air, and condenses back against cold surfaces. Evidence of these patterns of energy transfer are all around us, for example the heat radiating from electrical devices, or a liquid transforming to gas when boiling a kettle. Despite the ability to digitally monitor these energy patterns, the aim of conventional heating and ventilation systems is to mitigate localised changes in energy behaviour and to generate a standardised environment at the scale of a building (Addington and Schodek, 2005:64). Sensing and response systems are conventionally utilised to fully automate control of heating and ventilation, so that the interior climate can be maintained regardless of the weather outdoors. Maintaining ambient temperatures is energy intensive process that requires an input of heat during cold weather and the use of mechanised cooling systems during hot weather.

\subsection{The Responsive Environment}

An alternative approach is to engage directly with the responsive environment and use the pattern of energy flow as a design tool for interior or exterior spaces. Working with modelling systems such as thermal mapping, designers have produced new methods to re-imagine architectural space using the interior microclimate as the primary driver (Rahm 2006:118). In order to realise material systems that operate within this active and responsive design space new materials are required with the ability to sense and respond to environmental change. Here smart and programmable materials offer the potential to provide a dynamic interface between themselves and their surroundings: 
Whereas standard building materials are static in that they are intended to withstand building forces, smart materials are dynamic in that they behave in response to energy fields. (Addington \& Schodek, 2005:4)

The introduction of these materials provides the ability to generate localised interventions in response to the natural pattern of energy flow. This presents the opportunity to redefine an interior space from the perspective of discrete stimuli that change and adapt over time.

\subsection{Programmed Behaviour using Passive Responsive Materials}

Materials that respond intuitively to environmental stimuli enable designers to produce climate sensitive architectures that react in real time to environmental change. Passive responsive materials change their shape directly as a result of environmental stimuli. The stimulus could be chemical, thermal, or mechanical; however, these materials act directly with the stimulus, they do not require any electrical power. Two examples are thermobimetals and wood veneer. Thermobimetals are a composite of two different metals laminated together, which have different thermal expansion properties. When heat is applied the passive component expands at a lower coefficient of thermal expansion, and the active component resists expansion up to a higher coefficient of thermal expansion. As the two materials react differently the outcome is that the metal sheet bends (Ritter, 2007:53). A recent installation, Bloom exploits this shape change functionality at an architectural scale (Sung, in eds. Ng \& Patel, 2013:95). This piece, composed of 14,000 different tiles was designed to manage the thermal impact of solar energy; each tile would open and close in response to temperature changes generated by direct sunlight. The system operated autonomously in response to the changing weather conditions.

Whilst thermobimetal curls in one specific direction, it is possible to engineer natural materials to curl in different ways. Wood veneer curls when exposed to water. This is because wood is an anisotropic material and it swells and shrinks by different amounts in different directions. (Tsoumis, 1991:145). The way that it curls can be manipulated through the direction of cutting in relation to the direction of the grain. It is therefore possible to programme the material through the direction of cut. Wood veneer cut as a strip along the grain will bend top to bottom, cut against the grain will bend from the sides, whereas a diagonal cut across the grain will twist when exposed to water (Scott, 2015).

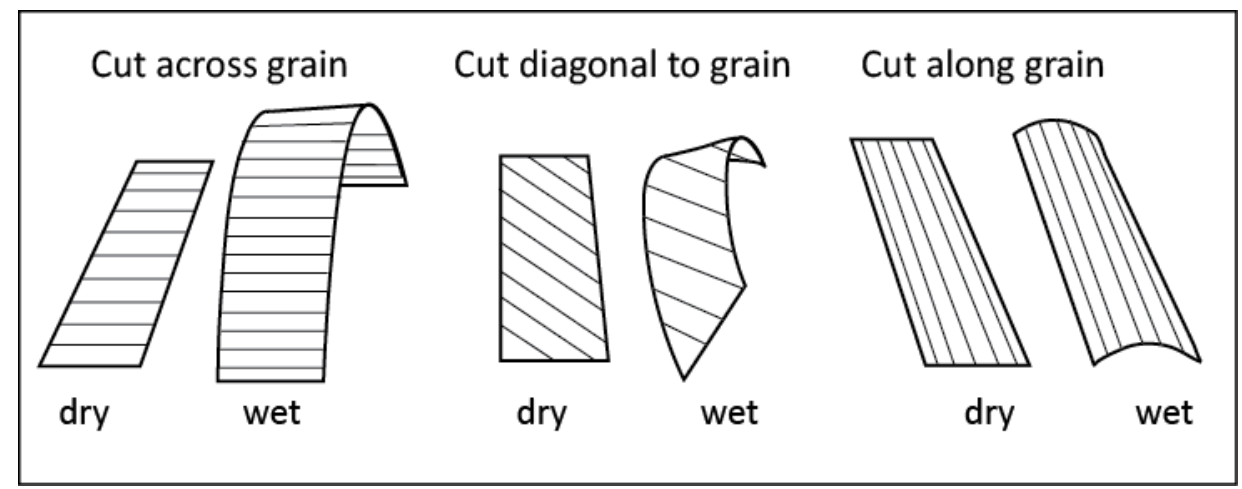

Figure 1 Illustration describing bending achieved when different cuts of veneer are exposed to water. Source: Scott.

The potential for veneer to produce environmental responsive architectures has produced a range of innovative outcomes. Techno-Naturology combines wood veneer with SMP to actuate a laser cut surface, producing heat activated shape change (Yan Ling, 2011:137), whereas Responsive Expansion (Maisonet and Smith, 2013:25-32) and Responsive Surface Structure II (Menges and Reichert, 2012:58) both utilise the moisture active properties of wood veneer in order to produce environmentally sensitive actuation. Wood veneer can be engineered to curl in specific ways by carefully controlling the way it is cut; the key consideration is the relationship between the structure of the material (the grain of wood, and direction of growth), and the geometries of cutting (the 
shape of the piece, and the angle of cut in relation to the structure of the material). This is significant as it demonstrates how a $100 \%$ natural material can be used to generate not only one, but a series of different shape change behaviours.

\section{Methodology}

Whilst innovation developing programmed behaviour using passive responsive materials has increased with progress in materials science and digital technologies, advances in biology research across a variety of scales from nano to macro has also provided a catalyst for the development of programmable material systems (Tibbits, 2017). In fact, nature presents an excellent model to inform research within this sector. The ability to sense and respond to changes in the environment is an essential characteristic of all living organisms. Plants are sensitive to a variety of changes in the environment and tropisms (directional movements) can be stimulated by light (phototropism), sunlight (heliotropism), water (hydrotropism) and chemicals (chemotropism) (Scott, P, 2008:161). As a design methodology biomimicry provides a systematic method to translate functional models from the natural world into effective, sustainable design solutions (Vincent, 2008:3140, Pawlyn, 2011:2, Bennyus, 1997:4).

\subsection{Biomimicry}

Biomimicry presents an opportunity to transform specific functions from nature into design. This paper focuses on analysis of sense and response systems in plants, in particular tissue structured to generate specific passive actuation in response to environmental changes. In order to apply the functionalities observed in natural materials in the design of programmable knitted fabrics, a method to formally abstract and translate the models is required. This methodology applies a direct model of biomimicry, using a problem based approach which begins with the questions of how to engineer shape change into the structure of a textile. The methodology (figure 2 ) is adapted from Knippers and Speck (2012).

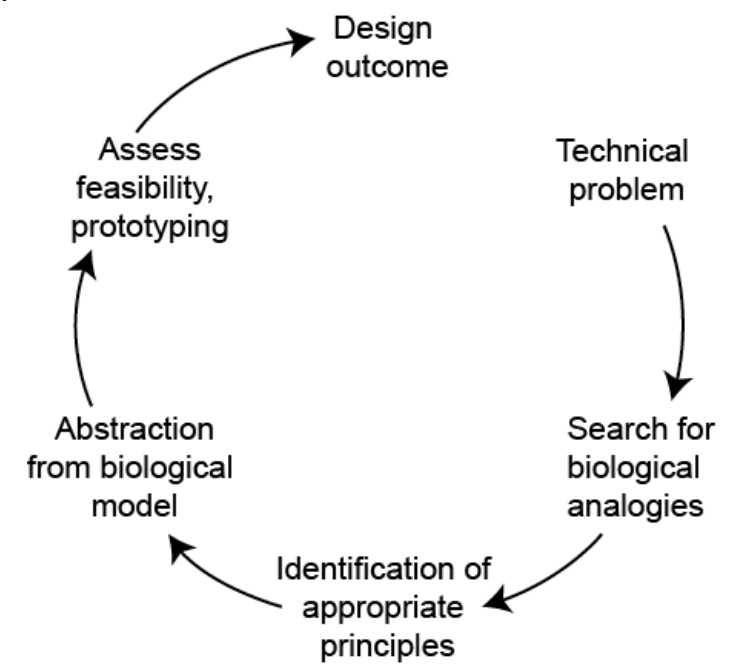

Figure 2 Biomimicry methodology using a problem based approach (adapted from Knippers and Speck, 2012:6, Figure 5). source: Scott.

In order to apply the methodology specific biological models are required to act as design principles to abstract into research, experimentation and prototyping. In this paper passive actuation systems in plants provide the biological analogies for application in textile design research.

\subsection{Actuation systems in plants}

As we have seen in the example of wood veneer, some plant materials have the ability to change in shape even when the material is no longer living. For example, pine cone scales are able to open and close repeatedly. The scales open in dry weather, revealing the seeds inside, and close in wet 
weather (Dawson et al, 1997:668). This action can be observed even after the pine cone has fallen from the tree, suggesting that the mechanism to control the shape change is inherent to the structure of the material itself.

This kind of passive actuation in plants is generated as a result of the composition of the material. It occurs where the structure of the material is composed in such a way that it is programmed to bend or coil when triggered by an external stimuli such as moisture or touch. Many passive movements are caused by the differential swelling and shrinking or specific parts of the plant tissues. This hygroscopic movement is used to control seed dispersal (Abraham and Elbaum, 2013:584). The movement is controlled by the differential structure of the plant cell wall. The plant cell is composed of stiff cellulose microfibrils embedded into a flexible matrix of hemicellulose and lignin. The contrasting mechanical properties produce a bilayer effect, controlled by the orientation of the cellulose microfibrils in the cell wall (Burgert and Fratzl, 2009: 1546).

To generate shape change, water is absorbed into the cell wall causing the tissue to expand. The flexible matrix swells with the influx of water and shrinks as it dries out; however the stiff cellulose fibrils do not swell and shrink to the same extent. As the two tissues are attached to one another, the way each material behaves is effected by the other material. The expansion is therefore anisotropic and causes a bending movement in the plant tissue.

These effects are generated across different scales within a pine cone scale; by mapping the impact of scales from nano to macro against those of a knitted fabric that the opportunity emerges to engineer shape change into the structure of a knitted fabric. Table 1 illustrates the key hierarchies and their role in the passive responsive system of the pine cone hygromorph as well as the opportunity that this presents for shape change in knitted fabric.

Table 1 Table Illustrates hierarchies observed in plant materials and pine cone hygromorph, and how this translates into requirements for shape change in textiles (Developed from Gibson, 2012 1-8, and Dawson et al, 1997:668).

\begin{tabular}{|c|c|c|}
\hline Hierarchy & Application to Pine cone Model & Requirement for moisture activated shape change \\
\hline $\begin{array}{l}\text { Biochemical } \\
\text { Cellulose }\end{array}$ & $\begin{array}{l}\text { Dimensional changes occur in pine } \\
\text { cone scale as water is absorbed. This } \\
\text { is regulated by different cell and } \\
\text { tissue structures. }\end{array}$ & Fibre : Fibres change dimension when water is absorbed. \\
\hline $\begin{array}{l}\text { Ultra- } \\
\text { structural } \\
\text { Cell Wall }\end{array}$ & $\begin{array}{l}\text { Variation in winding angle relative to } \\
\text { the long axis of the cell. High winding } \\
\text { angle allows fibres to expand and } \\
\text { contract (a). Low winding angle } \\
\text { prevents this (b). }\end{array}$ & Yarn: Winding angle alters dimensional changes. \\
\hline $\begin{array}{l}\text { Microscopic } \\
\text { Cells }\end{array}$ & $\begin{array}{l}\text { The pine cone scale is composed of } \\
\text { two types of cell: sclerids and } \\
\text { sclerenchyma fibres. }\end{array}$ & Knit stitch: Multiple unit (stitch) types required. \\
\hline $\begin{array}{l}\text { Macroscopic } \\
\text { Tissue }\end{array}$ & $\begin{array}{l}\text { Arrangement of sclerids and } \\
\text { sclerenchyma fibres allows scale to } \\
\text { open and close. As the sclerids } \\
\text { expand the scale is forced to bend due } \\
\text { to resistance from sclerenchyma } \\
\text { fibres. }\end{array}$ & $\begin{array}{l}\text { Fabric structure. Arrangement of different units form a } \\
\text { continuous material. }\end{array}$ \\
\hline
\end{tabular}




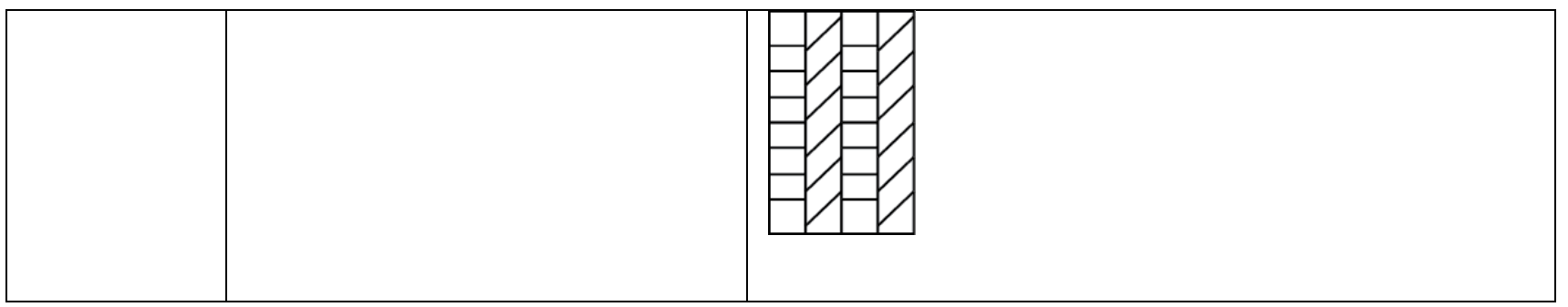

\section{Materials and Processes}

The hierarchical approach outlined above highlights specific opportunities to develop shape change behaviours. This section evaluates the components of knitted fabrics in order to identify how these principles can be implemented within knitted fabric design. The four hierarchies of biochemical, ultrastructural, microscopic and macroscopic translate into a knit fabric as fibres, yarns, knit stitch and fabric structure. Each section highlights how responsive behaviour can be engineered into the resultant fabric at the particular stage of construction. It is critical to note that whilst dimensional change in response to moisture can be observed at a fibre level, in order to control and manipulate the dimensional change into an overall shape change behaviour, careful control of the parameters of yarn, fabric and form are also required (Scott, 2015).

Yarns for knitting are composed of a mass of individual textile fibres twisted together. All fibres are long, fine and flexible; however their specific properties vary considerably depending on their chemical composition and physical structure (Wynne, 1997:1). All natural fibres have dynamic moisture absorption properties; fibres swell and increase in volume and density in the presence of moisture. These dimensional changes impact the yarns and fabrics made from them; changes in size, shape, stiffness, and permeability occur. This has a direct impact on their mechanical properties (Morton \& Hearle, 1986:159). As the fibres swell polymer chains are moved apart as the water molecules are absorbed. The swelling of fibres in water occurs at different rates in different directions. Swelling can be considered in terms of an increase in length (axial swelling), or an increase in diameter (transverse swelling) however fibres swell more across their diameter than along their length.

\section{All the moisture absorbing fibres show a large transverse swelling, but in the axial} direction swelling is very small, so that the swelling anisotropy is high (Morton \& Hearle, 1986:227).

The difference in the amount of swelling across the diameter of a fibre in comparison to along the length is critical. In the biomimetic model of the pine cone hygromorph, the transverse swelling of individual fibres causes the scale to open because of the arrangement of fibres within the scale (Fratzl \& Barth, 2009), and it is this principle can be transferred to knitted fabric design. However, it is critical to manipulate this anisotropic swelling in order to achieve shape change behaviours at the macro scale of the knitted fabric. To control of the dimensional swelling properties of fibres, it is necessary to consider the impact of both spinning processes in the construction of yarns as well as fabric structures and the overall fabric forms.

Whilst the orientation of fibres in a pine cone scale is determined through the growth of the material, fibre orientation in yarns is regulated during the spinning process. Here fibres are aligned and twisted together providing strength and flexibility to the resultant yarn. The yarns used for programmable knitting combine singles and folded yarns, which give contrasting balance within their structures. Singles yarns are naturally unbalanced because the twist in the yarn is always introduced in one direction only (referred to as s or z) (Wynne, 1997:59).

The amount of twist in a yarn also varies during yarn production, this is recoded as the turns per metre (tpm) for a given yarn depends on the fibre, count (a measure of the thickness of a yarn determined by the ratio of length to weight) and final application of the yarn. High twist yarns (also 
known as over-twist and crepe) have more twist inserted than standard twist yarns. Programmable knitting combines high twist and standard twist yarns; varying from 430tpm to 1180tpm. These yarns, predominately designed for weaving often snarl and untwist themselves, trying to achieve a more balanced state. These yarns are also prone to shrinkage because the fibres are compacted within the yarn (Taylor, 1999:172).

In order to develop programmable knitted fabrics, the configuration of knit stitches needs to be engineered to create the potential for 2D to 3D shape change. Knitted fabrics are composed of loops of yarn, and many of the characteristics of the fabric derive from the loop construction process. It is well known that knitted structures are highly extensible; in addition, knitted fabric have excellent deformation and recovery compared to other textiles (Spencer, 2001: 45).

Knitted fabric can be produced as flat-shaped pieces or as three-dimensional forms. Fabric shape can be altered by increasing or decreasing the number of stitches knitted in any course by transferring the individual knitted loops. Increasing the number of stitches at different points along a horizontal course changes the geometries of the fabric considerably. 2D, 3D and hyperbolic forms can be generated through varying both the number of needles knitting and the transfer points across a knitted course.
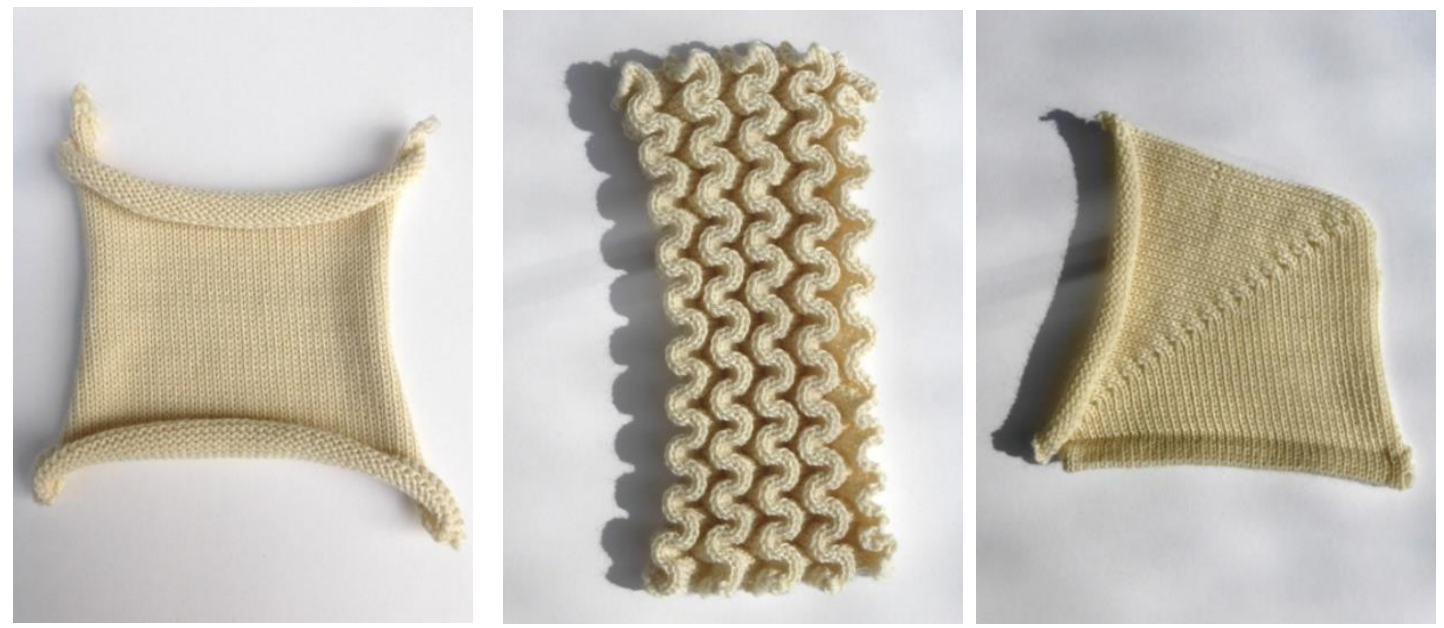

Figure 3 classes of weft knit fabric; plain knit, links/links and partial knit. source: Scott.

The knitting process generates particular behaviours that are consistent across any knitted fabric. For example, a plain knit fabric will always demonstrate the same curling behaviours (figure 3), because individual knitted loops are intermeshed in the same direction. For programmable knitting this directional behaviour is exploited in order to generate shape change behaviours. By manipulating the way that the plain fabric curls by changing the orientation of stitches using stitch transfer both complex geometries and programmed shape change can be engineered directly into the knitting structure (Scott, 2015). The manipulation of the curl of knit fabric is a fundamental principle for programmable knitting.

In addition to this the knitting process can be described as additive; fabric is generated on a stitch by stitch basis, providing the opportunity to combine multiple structures and geometries within one fabric. The flexibility of knitting also allows the fundamental properties of loop length, yarn type to be altered during knitting which makes the production of a knitted fabric extremely versatile.

Through analysis of the properties of natural fibres, yarns, fabric structure and form outlined above, a design system has been developed which produces shape-changing actuation using only natural materials and conventional knit technologies.

\section{Design Application: Programmable Knitting}

To analyse the success of the biomimicry methodology an interior installation piece, Skew (Scott, 2014) is evaluated. Designed and manufactured using Shima Seiki CNC knit technologies, Skew, is a 
$1 \mathrm{~m} \times 2 \mathrm{~m}$ interior panel combining structural patterning at different scales across the full dimensions of the piece.

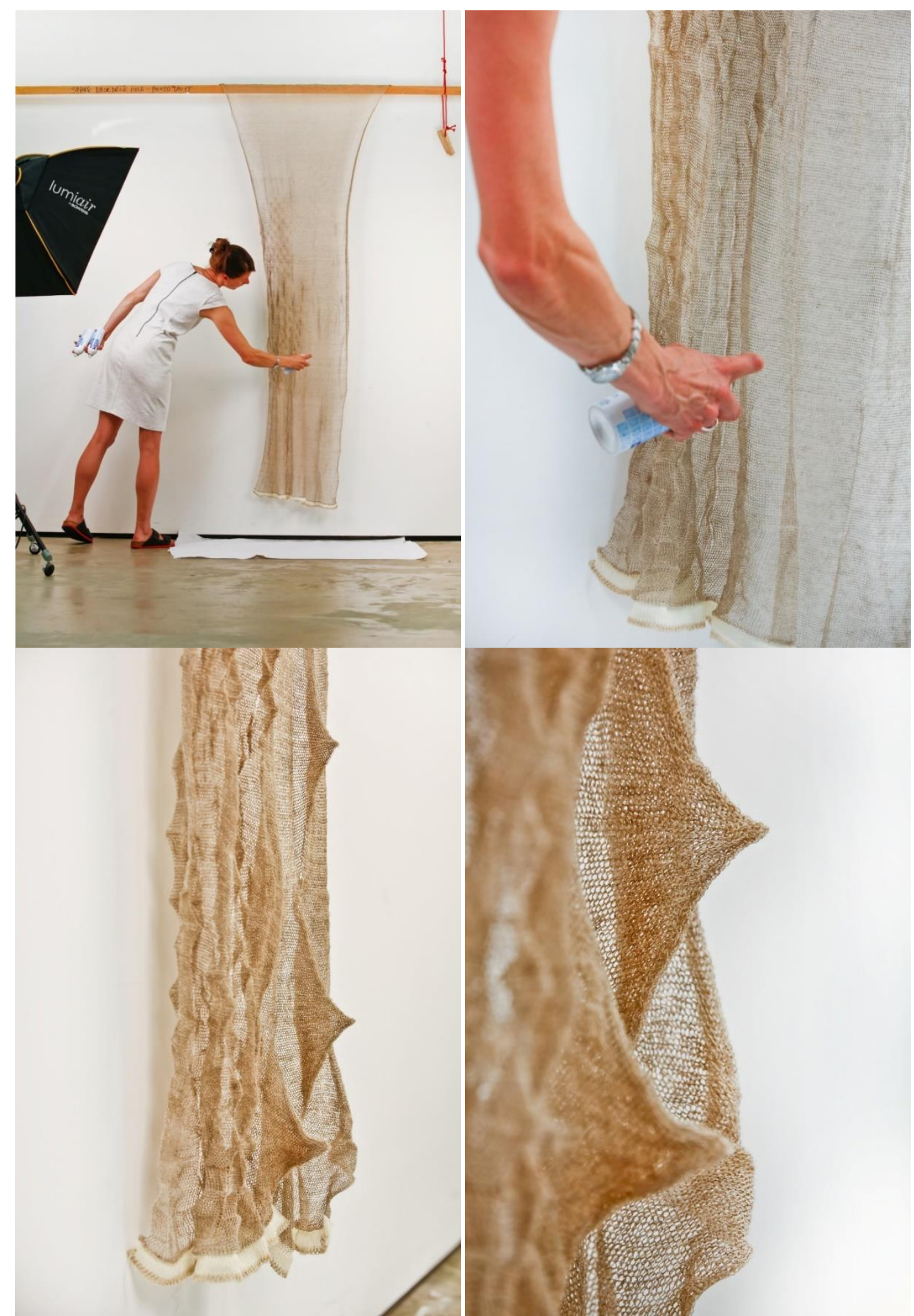

Figure 4 Skew: Montage of images illustrating the process of shape change behaviour. source: Scott. 

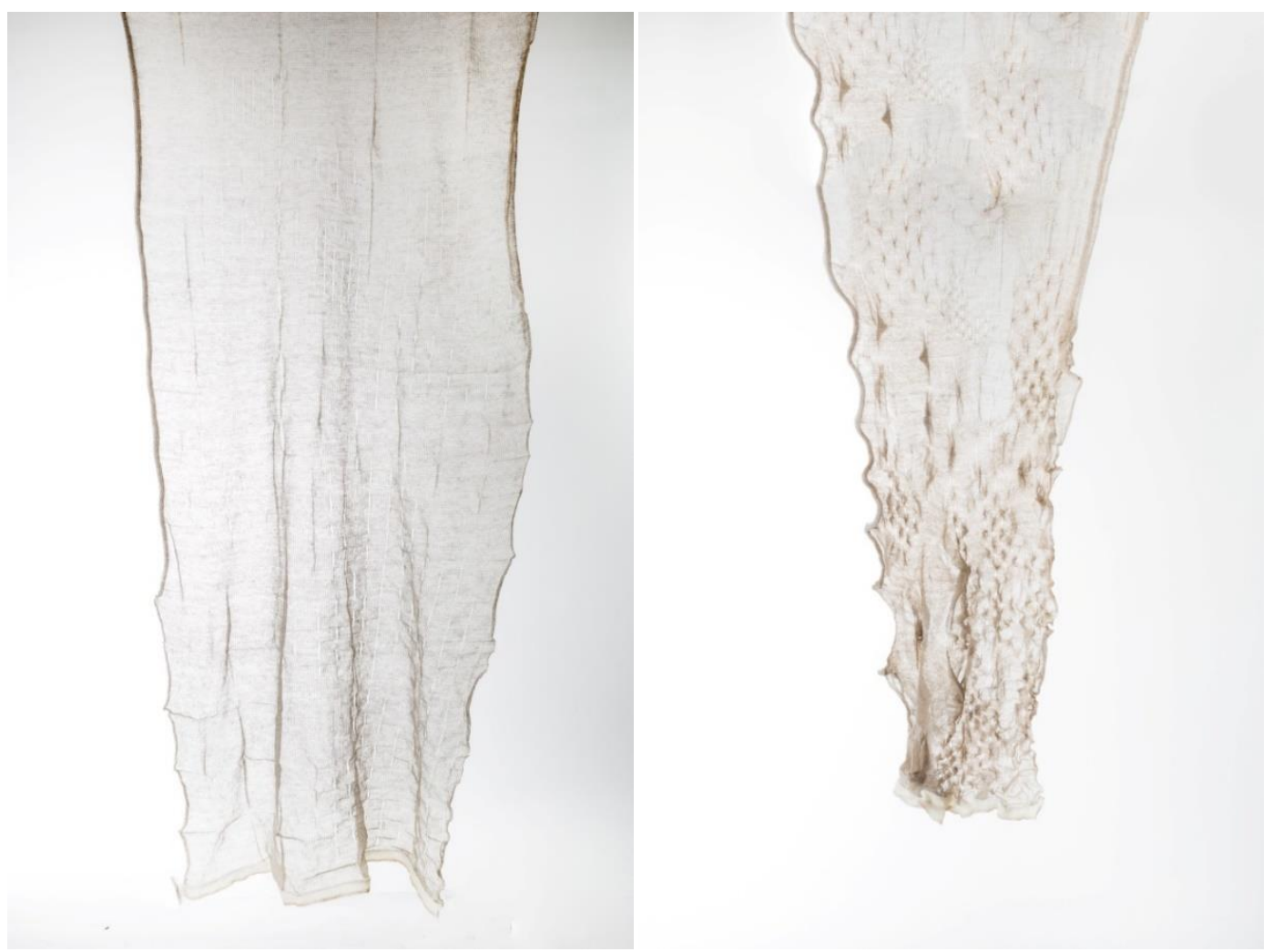

Figure 5 Skew: Before Actuation source: Scott.

Figure 6 Skew: After Actuation source: Scott.

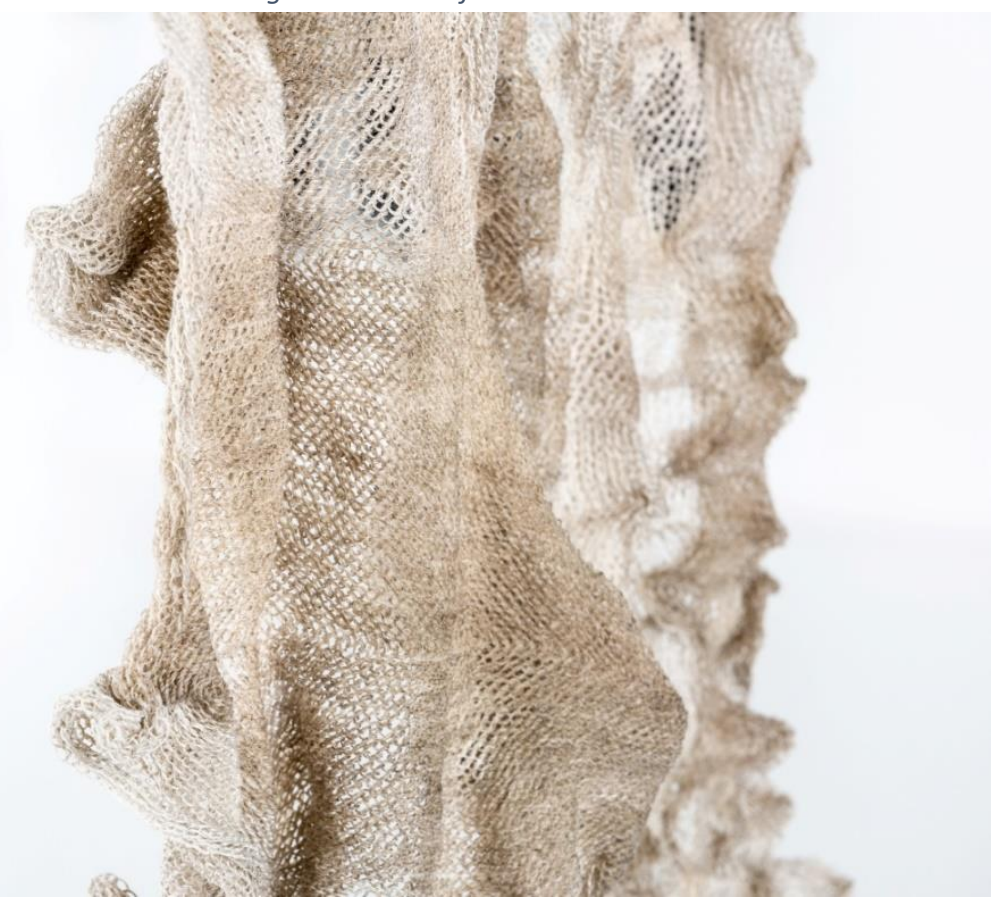

Figure 7 Skew: detail of 3D profile showing shape change at edges and different scales of patterning. source: Scott.

\subsection{Fabric Construction Process}

The work was programmed using the SDS1Apex system, and manufactured on a Shima Seiki NSSG $5 g g$ knitting machine. A major advantage of programming materials using the knit production processes is that they are constructed using yarns with standard properties required for knitting (good flexibility, strength and elastic recovery (Spencer,2001:4)), so the fabrics can be manufactured using a range of knitting technologies. This piece is composed of $1 / 24 \mathrm{~nm} s$ twist linen. The capabilities of the Shima Seiki programming system allow unlimited stitch transfer sequences across 
the full dimensions of the fabric which would be difficult and time consuming to achieve on hand operated equipment. Fabric width is determined by the width of the needle bed; and control is provided through computerised takedown and tensioning systems. This provides a mechanism to regulate fabric production and minimise faults during knitting.

\subsection{Analysis}

Skew is composed of a complex configuration of knit stitches organised into links/links patterns across a variety of scales. The smallest scale is five wales and five courses of face stitches opposing five wales and five courses of reverse stitches in a links/links configuration (5x5). Across the fabric the scale increases: $10 \times 10,25 \times 25$ and $50 \times 50$. The intention of the fabric design was to explore what happens when scales interact and whether the pace of shape change could vary dependant on the size of the repeats. It was anticipated that smaller repeats $(5 \times 5)$ would produce a rippling effect across the fabric on actuation whereas larger repeats would emerge as 2D to 3D forms across the fabric length.

The fabric was tested as a hanging panel, actuated using a cold water spray. In machine state the fabric hangs flat. Whilst it is possible to identify front bed and back bed stitches on the fabric, the repeats appear as a pattern of squares and there is minimal disruption to the smooth surface (figure 5). On actuation spiral peaks of different sizes form all over the fabric. The spiral peaks vary in height from $1 \mathrm{~cm}(10 \times 10)$ to $7 \mathrm{~cm}(50 \times 50)$. This causes an overall change in dimensions; the fabric lifts up from the bottom and shrinks in at the sides (figure 6). The edges of the fabric also change; in machine state the fabric is rectangular, however on actuation the edges shear to produce zigzags (figure 7).

On application of moisture the fabric instantly actuates as water is absorbed. There is a significant difference in the speed of actuation for areas with smaller repeats (10x10), transforming from 2D to $3 \mathrm{D}$ in three seconds whereas larger areas $(50 \times 50)$ transform in ten seconds. This produces a rippling effect and a sense of motion across the whole piece in addition to the overall change in form and dimensions.

When the fabric is hanging, shape change is most dramatic in the bottom half of the fabric (figure 6). This is visible as an overall effect as the fabric distorts and appears to lift up. There are two reasons for this; firstly the top of the fabric is secured in position (for exhibition a Perspex rod was used to hang the fabric) preventing movement at the top of the fabric. Secondly as moisture is absorbed, the linen becomes heavier. It is therefore harder for the shape change to occur at the top of the fabric as it is weighed down by the bottom (figure 6). Despite this, the overall change in dimensions is significant; the fabric measures $65 \mathrm{~cm} \times 220 \mathrm{~cm}$ in a dry state, and $30 \mathrm{~cm} \times 190 \mathrm{~cm}$ on actuation (Width measurement $20 \mathrm{~cm}$ from bottom). Spiral peaks form all over the fabric. These vary in height from $1 \mathrm{~cm}$ to $7 \mathrm{~cm}$. Shape change is also reversible. As the fabric dries it returns to a flat state and can be re-actuated with water.

Of particular interest within this piece are the edges which distort to form a zigzag up the fabric, and the sections where different scales of patterning interact (figure 7). In some places the disruption in the pattern counteracts the 3D transformation and the fabric does not change shape, however in other areas new 3D shapes are generated.

This piece was first exhibited in March 2015, as part of RTD2015 Research Through Design, at the Microsoft Research Centre in Cambridge. This event combined a conference and exhibition. During discussions of the work during a testing session the conversation discussed potential applications for the technology as a sensing system within the environment. Delegates commented on the potential for the material system, illustrated through Skew, to define an alternative rhythm or timeframe for architectural space (Author,2015), The suggestion is that shape change could be representative of the changing environmental conditions within the interior environment. This would provide a direct connection to microclimates that emerge within an interior space as a result of both the natural energy patterns in a space and human interventions. Examples discussed included utilising 
programmable knitting to monitor moisture in bathrooms and kitchens where levels vary considerably during the day and night.

\section{Conclusions}

The context of the responsive environment is critical to this design research; it provides an active design space which demands a material system that can directly engage with changing environmental conditions over time. In this research the design space is explored in relation to humidity and moisture levels, using textile fibres with the ability to swell, changing in dimensions when exposed to high levels of moisture. Other moisture responsive are identified including responsive wood architectures (Reichert and Menges, 2015) and superabsorbent polymer composites (Tibbits, 2014), however what is unique about programmable knitting the way that the dimensional changes at the level of the fibre are translated into an actuation motion at the level of the fabric.

The application of a textile logic in the development of programmable materials adds significant opportunity for materials development. The textile system introduces three or four levels of complexity in relation to the hierarchical system. Here shape change is determined through the relationship of fibre, yarn, fabric structure and fabric form, and changes at any level of hierarchy will produce different results in the actuated form. Each of these textile components provide the opportunity to engineer the dimensional change in an individual fibre into a macro scale shape change. Directionality introduced when yarns are spun offers the potential to translate the individual dimensional changes at the level of the fibre into an actuation motion at the level of the fabric. The infinite variety of configurations of knitted stitches provides a huge variation in the resulting shape change achievable. Programmable knitting offers not only a variety of shape change responses articulated through 2D to 3D transformations in fabrics, but a variety of speeds of responses; each fabric articulates an alternative timeframe for the responsive environment dependant on the constituent materials and configurations of knitted stitches.

In conclusion this work offers a series of examples which could be used for further research into programmable materials. Firstly, the work demonstrates that additional functionality can be designed into materials using conventional manufacturing processes such as knitting. This presents an interesting question for research that has been traditionally led through advances in materials science and technology. Instead this work provides a precedent for re-evaluating the potential of current technologies from a new perspective.

Secondly, advanced manufacturing systems for the design of programmable materials, (such as 3D print) use additive manufacturing process which, like knitting design materials from the bottom up. This presents huge opportunities to reflect the way that natural materials grow, embedding complexity into the structure of the material itself. The model of structural hierarchy derived from plant materials is significant as an example of how to analyse behaviour at individual scales across multiple hierarchies, and analysis of materials at the microscale of an individual fibre provides insight into how a material could behave at the macroscale of a fabric.

Finally, the application of biomimicry motivates inherently sustainable design choices. In this research material selection is exclusively $100 \%$ biodegradable natural fibres and by programming shape change into the fabric itself there are no additional components required for shape change to occur. Smart synthetics and electronic control have become redundant within this programmed textile system. This is the ultimate challenge working within the context of the responsive environment; the development of sustainable tools to maintain and support interactions with the environment at a material scale.

\section{References}

Abraham, Y. and Elbaum, R. (2013). Hygroscopic movements in Geraniaceae: the structural variations that are responsible for coiling or bending. New Phytologist. 199, pp.584-594. 
Addington, M. and Schodek, D. (2005). Smart Materials and Technologies for the architecture and design professions. Oxford: Elsevier.

Benyus, J. (1997). Biomimicry: Innovation Inspired By Nature. New York: Perennial.

Burgert, I. and Fratzl, P. (2009). Actuation systems in plants as prototypes for bioinspired devices. Phil. Trans. R. Soc. A. 367, pp.1541-1557.

Correa, D. \& Menges, A (2017) Fused Filament Fabrication for Multi-kinematic State Climate-Responsive Aperture, Fabricate, UCL, 191-195.

Dawson, C., Vincent, J. and Rocca, A.M. (1997). How Pine Cones Open Up. Nature. 390, p.668.

Eadie, L. and Ghosh, T. (2011). Biomimicry in Textiles, Past, Present and Potential, an Overview. J.R.Soc Interface. 59(8): pp.761-775.

Fratzl , P. and Barth, F.G. (2009). Biomaterial systems for mechanosensing and actuation. Nature. 462, pp.442448.

Gibson, L. (2012). The hierarchical structure and mechanics of plant materials. J.R.Soc. Interface. 9(76), pp.1-18.

Knippers, J. and Speck, T. (2012). Design and construction principles in nature and architecture. Biomimetics and Bioinspiration. 7(1), pp.1-10.

Maisonet, C. and Smith, A. (2013). Responsive Expansion. In: Proceedings of ACADIA2013, Adaptive Architecture, 21-27 October 2013, Waterloo. Canada: Riverside Architectural Press, pp.25-32.

Menges, A. and Reichert, S. (2012). Material Capacity: Embodied Responsiveness, AD. 82(2), pp.53-59.

Morton, W.E. and Hearle, J.W.S. (1986). Physical Properties of Textile Fibres. Manchester: Textile Institute.

Ng, R. and Patel, S. (2013). Performative Materials in Architecture and Design. Bristol: Intellect.

Pawlyn, M. (2011). Biomimcry in Architecture. London: RIBA Publishing.

Rahm, P. (2012). Meteorological Elements. [Online]. 12 April, The University of Pennsylvania, Pennsylvania. [Accessed 25 March 2014]. Available from: http://www.design.upenn.edu/news/video-philippe-rahmslecture-now-available

Reichert, S. Menges, A, \& Correa, D. (2015) Meterosensitive Architecture: Biomimetic Building Skins based on Materially Embedded and Hygroscopically Enabled Responsiveness, Computer Aided- Design, 60 50-69.

Ritter, A. (2007). Smart materials in architecture, interior architecture and design. Basel- Boston-Berlin: Birkhauser.

Scott, J. (2015). Programmable Knitting, PhD Thesis, The University of the Arts, London.

Scott, J. (2015). Mutate: The Evolution of a Responsive Knit Design System. Proceedings of Research through Design, Microsoft Research Centre, http://figshare.com/articles/RTD2015_05_Mutate_The

Evolution_of_a_Responsive_Knit_Design_System/1327974

Scott, P. (2008). Physiology and Behaviour of Plants. UK: Wiley.

Spencer, D.J. (2001). Knitting Technology. Cambridge: Woodhead.

Taylor, M.A. (1999). Technology of Textile Properties. London: Forbes.

Tibbits, S. (2014). 4D Printing. AD 84(1), pp.117-121.

Tibbits, S. (2017). Active Matter, MIT Press, Boston

Tsoumis, G. (1991). Science and Technology of Wood. London: Chapman and Hall.

Vincent, J. (2008). Biomimetic Materials. J. Mater. Res. 23(12), pp.3140-3146.

Wynne, A. (1997). Textiles. London: Macmillan.

Yan Ling, E. (2013). Techno-naturology. In: Dieffenbacher, F. Fashion Thinking: Creative Approaches to the Design Process. London: AVA Publishing, pp.133-137.

About the Author:

Jane Scott is a UK based designer and academic working at the intersection of biomimicry, programmable materials, textile design and technology. As a knit specialist her research challenges the established understanding of smart materials; applying principles derived from plant biology to the development of programmable and environmentally responsive textiles. Her work has been presented internationally at conferences and exhibitions including Massachusetts Institute of Technology, Make/Shift (Craft Council), the Microsoft Research Centre, and The London Design Festival. In 2016 she received the 2016 Autodesk ACADIA Emerging Research Award (projects category) for her work Programmable Knitting. 